\section{Doxorubicin Effect on Myocardial Metabolism as a Prerequisite for Subsequent Development of Cardiac Toxicity: Are There Unsuspected Confounders?}

TO THE EDITOR: We have enthusiastically read the meticulous and well-conducted retrospective study by Bauckneht et al. published in the October issue of The Journal of Nuclear Medicine (1). The authors assessed the role of ${ }^{18} \mathrm{~F}$-FDG PET/CT in the prediction of doxorubicin cardiac toxicity in 69 patients treated with a chemotherapy regimen for Hodgkin lymphoma (HL); furthermore, they investigated the possible dose-dependent nature of doxorubicin toxicity in 15 athymic mice and concluded that in patients undergoing doxorubicin administration, not only is myocardial ${ }^{18} \mathrm{~F}$-FDG uptake increased but also that low ${ }^{18} \mathrm{~F}-\mathrm{FDG}$ uptake before chemotherapy may predict the development of cardiac toxicity.

Myocardial cells protect themselves from hypoxic state, reducing contractile function with the downregulation of hypoxia and mitochondrial oxidative metabolism through the "glucosefatty acid cycle" (2), and ${ }^{18}$ F-FDG uptake may be a useful tool to identify a myocardial metabolic switch subsequent to cellular damage (3).

Several published retrospective and preclinical studies, some already cited by Bauckneht et al. $(4,5)$, suggest the opportunity of investigating the correlation between changes in myocardial ${ }^{18} \mathrm{~F}$ FDG uptake in pre- and postchemotherapy or radiotherapy evaluation and subsequent development of cardiac toxicity $(6,7)$.

We also observed in a preliminary study that cardiac uptake of ${ }^{18} \mathrm{~F}-\mathrm{FDG}$ could increase during chemotherapy (8). Our first hypothesis was that in patients undergoing chemotherapy some elements may modify the variable avidity for glucose (9) and shift the myocardial metabolism from $\beta$-oxidation of fatty acids to glycolysis, also considering the possible role of steroids (for iatrogenic hyperglycemia) and granulocyte colony-stimulating factor (for insulinlike effects). We tested our hypothesis retrospectively in a group of HL patients $(n=24)$ treated with a regimen of adriamycin, bleomycin, vincristine, dacarbazine (ABVD) plus $20 \mathrm{mg}$ of dexamethasone, excluding patients with antecedent cardiovascular disease, diabetes, and previous chemotherapy or mediastinal irradiation and identifying 10 patients who received from 50 to $575 \mathrm{mg}$ of steroids additional to the standard (extra-steroids group).

All patients underwent ${ }^{18} \mathrm{~F}-\mathrm{FDG} \mathrm{PET} / \mathrm{CT}$ at staging, interim, and final evaluation: no significant differences were found between different scans in patients' body weight and glycemia levels at ${ }^{18} \mathrm{~F}$-FDG injection. We observed also an incremental trend in cardiac $\mathrm{SUV}_{\max }$ at staging, interim, and final evaluation, and we found a significant association between ${ }^{18} \mathrm{~F}$-FDG uptake and extrasteroid administration $(P=0.005)$, suggesting a strong, independent (and possibly transient) correlation between administration of extra steroids and this phenomenon.

The correlation between cardiac toxicity after chemotherapy, in particular after anthracycline administration, or radiotherapy and myocardial ${ }^{18} \mathrm{~F}$-FDG uptake is a current study argument.

Given the aforementioned data, it is our opinion that ${ }^{18} \mathrm{~F}-\mathrm{FDG}$ PET/CT may be a useful biomarker of cardiac toxicity, but only after first clarifying the role of other factors that may occur as confounders in ${ }^{18}$ F-FDG uptake, such as steroid administration.

\section{REFERENCES}

1. Bauckneht M, Ferrarazzo G, Fiz F, et al. Doxorubicin effect on myocardial metabolism as a prerequisite for subsequent development of cardiac toxicity: a translational ${ }^{18}$ F-FDG PET/CT observation. J Nucl Med. 2017;58:1638-1645.

2. Randle PJ, Garland PB, Hales CN, Newsholme EA. The glucose fatty-acid cycle: its role in insulin sensitivity and the metabolic disturbances of diabetes mellitus. Lancet. 1963;1:785-789.

3. Dilsizian V, Narula J. Atlas of Nuclear Cardiology. 4th ed. New York, NY: Springer; 2013.

4. Borde C, Kand P, Basu S. Enhanced myocardial fluorodeoxyglucose uptake following adriamycin-based therapy: evidence of early chemotherapeutic cardiotoxicity? World J Radiol. 2012;4:220-223.

5. Gorla AK, Sood A, Prakash G, Parmar M, Mittal BR. Substantial increase in myocardial FDG uptake on interim PET/CT may be an early sign of adriamycininduced cardiotoxicity. Clin Nucl Med. 2016;41:462-463.

6. Evans JD, Gomez DR, Chang JY, et al. Cardiac ${ }^{18} \mathrm{~F}$-fluorodeoxyglucose uptake on positron emission tomography after thoracic stereotactic body radiation therapy. Radiother Oncol. 2013;109:82-88.

7. Basu S, Borde C, Kand P. Increasing cardiac ${ }^{18}$ F-fluorodeoxyglucose (FDG) uptake on PET-CT as a biomarker for cardiotoxicity of chemoradiotherapy in cancer: a myth or a reality? Radiother Oncol. 2014;112:451-452.

8. Parente A, Finessi M, Nicolotti D, et al. Cardiac FDG uptake increases after ABVD (adryamicine, bleomycine, vincristine, dacarbazine) and support therapy with steroids and G-CSF: an observation on 24 patients with newly-diagnosed Hodgkin's disease. Eur J Nucl Med Mol Imaging. 2012;39(suppl 2):S469.

9. Osborne MT, Hulten EA, Venkatesh LM, et al. Patient preparation for cardiac fluorine-18 fluorodeoxyglucose positron emission tomography imaging of inflammation. J Nucl Cardiol. 2017;24:86-99.

Monica Finessi*
Daniele G. Nicolotti
Gianni Bisi
Désirée Deandreis
*Division of Nuclear Medicine
Department of Medical Sciences
AOU Città della Salute e della Scienza
University of Turin, Italy
Corso Dogliotti 14, 10126 Torino
E-mail: finessimonica@ @mail.com

Published online Jan. 11, 2018.

DOI: 10.2967/jnumed.117.205856

REPLY: We are grateful to Finessi et al. for their letter, which raises a crucial topic. We agree that many possible confounding factors may challenge the interplay between anthracycline-induced cardiotoxicity and myocardial ${ }^{18} \mathrm{~F}-\mathrm{FDG}$ accumulation. Among them, steroids might profoundly interfere with myocardial metabolism, increasing insulin resistance, reducing free fatty acid serum levels, and consequently potentially affecting myocardial ${ }^{18} \mathrm{~F}$-FDG uptake (1).

On the other hand, the relevance of corticosteroids nicely fits with our hypothesis about the existence of a peculiar ${ }^{18} \mathrm{~F}-\mathrm{FDG}$ metabolic pathway located in the endoplasmic reticulum and regulated by hexose-6-phosphate dehydrogenase (H6PD) (2). This enzyme represents the unique reticular source of the reduced nicotinamide adenine dinucleotide phosphate (NADPH) moieties needed for cortisone activation $(3,4)$ in the same endoplasmic reticulum by 11 betahydroxysteroid dehydrogenase type 1 (11ß-HSD1). In this line, the increased use of NADPH reductive power caused by the administration of pharmacologic cortisone doses might "transiently" increase H6PD activity and thus myocardial ${ }^{18}$ F-FDG uptake. However, this variable should have played a minor role in our main observation (5) for the following reasons. 
First, in our experimental animal model, in which steroids were not administered, DXR dose-dependently increased myocardial ${ }^{18} \mathrm{~F}-\mathrm{FDG}$ accumulation. Second, as suggested by Finessi et al. in their previous study (6), the extra-steroid administration might be a strong, independent thought "transient" variable able to affect myocardial ${ }^{18} \mathrm{~F}-\mathrm{FDG}$ accumulation. However, in our retrospective analysis, left-ventricular (LV) SUV was significantly increased in DXR-treated patients with respect to controls at the third PET study but remained persistently elevated 6 mo after chemotherapy (and eventually extra-steroid) discontinuation. Finally, and more importantly, when we focused on adriamycin, bleomycin, vincristine, dacarbazine-treated patients who accepted to undergo a later clinical evaluation, the occurrence of DXR-induced cardiotoxicity was significantly related to lower values of LV-SUV at baseline, in which patients did not received any therapy.

Altogether these observations seem to indicate that whatever its degree, the effect of cortisone therapy should have been transient and independent from baseline condition.

\section{REFERENCES}

1. Manabe O, Yoshinaga K, Ohira H, et al. Steroid therapy affects the cardiac metabolic shift and requires the careful attention in evaluation of the cardiac sarcoidosis with FDG PET/CT [abstract]. J Nucl Med. 2016;57(suppl 2):178.

2. Marini C, Ravera S, Buschiazzo A, et al. Discovery of a novel glucose metabolism in cancer: the role of endoplasmic reticulum beyond glycolysis and pentose phosphate shunt. Sci Rep. 2016;6:25092.

3. Bujalska IJ, Draper N, Michailidou Z, et al. Hexose-6-phosphate dehydrogenase confers oxo-reductase activity upon 11 beta-hydroxysteroid dehydrogenase type 1 . $J$ Mol Endocrinol. 2005;34:675-684.

4. Cooper MS, Rabbitt EH, Goddard PE, Bartlett WA, Hewison M, Stewart PM. Osteoblastic 11beta-hydroxysteroid dehydrogenase type 1 activity increases with age and glucocorticoid exposure. J Bone Miner Res. 2002;17:979-986.

5. Bauckneht M, Ferrarazzo G, Fiz F, et al. Doxorubicin effect on myocardial metabolism as a prerequisite for subsequent development of cardiac toxicity: a translational ${ }^{18}$ F-FDG PET/CT observation. J Nucl Med. 2017;58:1638-1645.

6. Parente A, Finessi M, Nicolotti D, et al. Cardiac FDG uptake increases after ABVD (Adryamicine, Bleomycine, Vincristine, Dacarbazine) and support therapy with steroids and G-CSF: an observation on 24 patients with newly-diagnosed Hodgkin's disease. Eur J Nucl Med Mol Imaging. 2012;39:S469.

Gianmario Sambuceti* Silvia Morbelli Vanessa Cossu Cecilia Marini Matteo Bauckneht *University of Genova Largo R. Benzi 10 16132 Genova, Italy E-mail:sambuceti@unige.it

Published online Jan. 18, 2018. DOI: 10.2967/jnumed.117.206797

\section{Staging, Restaging, and Treatment Response Assessment in Lymphomas: What We Should Know}

TO THE EDITOR: We read with interest the review published by Jadvar and colleagues (1) who highlight the main indications of ${ }^{18} \mathrm{~F}-\mathrm{FDG} \mathrm{PET} / \mathrm{CT}$ for restaging and treatment response assessment in oncology. They point out its clinical impact in 7 of the most commons cancers. However, we think it is necessary to give additional information on the role of ${ }^{18} \mathrm{~F}$-FDG PET/CT in lymphomas and to draw the attention of the readers to the most relevant papers in the field. Indeed, on the basis of the results of important studies, PET/CT has changed the management of many subtypes of lymphoma and we would like herein to address some key points.

Lymphomas are a heterogeneous group of tumors encompassing numerous entities with different prognosis and treatment. Lymphomas were usually classified as ${ }^{18} \mathrm{~F}-\mathrm{FDG}$-avid or nonavid, depending of their subtypes. The role of ${ }^{18} \mathrm{~F}-\mathrm{FDG}$ PET/CT, initially limited to some of the ${ }^{18} \mathrm{~F}-\mathrm{FDG}$-avid subtypes (Hodgkin lymphoma [HL], diffuse large B cell [DLBCL], and follicular lymphomas), is now recognized for the clear majority of lymphomas and has been recently demonstrated for $\mathrm{T}$ cell lymphoma, mantle cell lymphoma, and plasmablastic lymphomas. It should be emphasized that, among the main roles of ${ }^{18} \mathrm{~F}-\mathrm{FDG}$ PET/CT at staging, PET is now recommended as a noninvasive technique for the detection of bone marrow involvement in HL and DLBCL and has replaced the invasive bone marrow biopsy in HL and in some cases in DLBCL (2). This recommendation has improved the quality of a patient's life. The second point is the role of ${ }^{18} \mathrm{~F}$ FDG PET/CT in follicular lymphoma, where it has shown capabilities (3) in identifying the tumor site of transformation and guiding biopsy for diagnosis. For staging, it is important to underline that for the ${ }^{18} \mathrm{~F}-\mathrm{FDG}$-avid lymphoma there is no more proven advantage than to perform separate contrast-enhanced CT because ${ }^{18}$ F-FDG PET/CT has a much better sensitivity.

Excellent general reviews and consensus papers have documented the indications of ${ }^{18} \mathrm{~F}-\mathrm{FDG}$ PET/CT according to each clinical challenge for each subtype of lymphoma. Therefore, we will mainly focus on the response assessment to treatment and will report on the main studies demonstrating the usefulness of interim PET-guided therapy.

Lymphomas are certainly the oncologic application for which ${ }^{18}$ F-FDG PET/CT provided the most significant improvement compared with conventional imaging. ${ }^{18} \mathrm{~F}-\mathrm{FDG}$ PET/CT allows a better identification of treatment failure and identifies patients who are deemed to benefit from salvage therapy. This evaluation at the end of the first line of treatment has been standardized on the basis of a Deauville 5-point scale and more recently reshaped in the Lugano classification (2). Compared with conventional imaging, ${ }^{18}$ F-FDG PET/CT provides a more reliable evaluation (removing the CT unconfirmed responses) and a better specificity in response evaluation, and leads to a more accurate identification of nonresponder patients with a strong prognostic impact. This high clinical value has been documented in large series for the main common lymphomas such as HL, DLBCL, and follicular lymphoma and was also validated in cost-effectiveness studies (4-6).

Large prospective trials, especially in HL, have shown that an early evaluation of treatment response using interim PET/CT after 2 cycles of chemotherapy (PET2) could be used to adapt treatment strategy. The H10 trial for early-stage HLs has shown that PETdriven chemointensification (adriamycin, bleomycin, vincristine, dacarbazine [ABVD] to BEACOPP regimen) of PET2-positive patients significantly enhances the progression-free survival (7). Similar data have been shown for advanced HL in several trials in which interim PET2-negative patients followed the initial ABVD treatment whereas the therapy regimen for PET2-positive patients was intensified with BEACOPP (8). This interim PET-personalized 